\title{
Prevalence of near-death experiences in people with and without REM sleep intrusion
}

\author{
Daniel Kondziella ${ }^{\text {Corresp., 1, 2, } 3}$, Jens P. Dreier ${ }^{4,5,6,7,8}$, Markus Harboe Olsen ${ }^{9}$ \\ ${ }^{1}$ Department of Neurology, Rigshospitalet, University of Copenhagen, Copenhagen, Denmark \\ 2 Faculty of Health Sciences and Medicine, University of Copenhagen, Copenhagen, Denmark \\ 3 Department of Neuroscience, Norwegian University of Technology and Science, Trondheim, Norway \\ 4 Center for Stroke Research Berlin, Charité Charité - Universitätsmedizin Berlin, corporate member of Freie Universität Berlin, Humboldt-Universität zu \\ Berlin, and Berlin Institute of Health, Berlin, Germany \\ 5 Department of Neurology, Charité Charité - Universitätsmedizin Berlin, corporate member of Freie Universität Berlin, Humboldt-Universität zu Berlin, \\ and Berlin Institute of Health, Berlin, Germany \\ 6 \\ 6 Department of Experimental Neurology, Charité Charité - Universitätsmedizin Berlin, corporate member of Freie Universität Berlin, Humboldt-Universität \\ zu Berlin, and Berlin Institute of Health, Berlin, Germany \\ 7 Bernstein Center for Computational Neuroscience Berlin, Berlin, Germany \\ 8 Einstein Center for Neurosciences Berlin, Berlin, Germany \\ 9 Department of Neuroanesthesiology, Rigshospitalet, University of Copenhagen, Copenhagen, Denmark \\ Corresponding Author: Daniel Kondziella \\ Email address: daniel_kondziella@yahoo.com
}

BACKGROUND: The origin and prevalence of near-death experiences are unknown. A recent study suggested a link with REM sleep intrusion but was criticized for its selection of control participants. We therefore assessed the association of REM intrusion and near-death experiences with different methods.

METHODS: Using a crowd-sourcing platform, we recruited 1034 lay people from 35 countries to investigate the prevalence of near-death experiences and self-reported REM sleep intrusion. Reports were validated using the Greyson Near-Death Experiences Scale (GNDES) with $\geq 7$ points as cut-off for near-death experiences.

RESULTS: Near-death experiences were reported by 106 of 1034 participants (10\%; Cl 95\% 8.5-12\%). Evidence of REM intrusion was more common in people with near-death experiences $(n=50 / 106 ; 47 \%)$ than in people with experiences with 6 points or less on the GNDES ( $n=47 / 183 ; 26 \%$ ) or in those without such experiences $(n=107 / 744 ; 14 \% ; p=<0.0001)$. Following multivariate regression analysis to adjust for age, gender, place of residence, employment and perceived danger, this association remained highly significant; people with REM intrusion were more likely to exhibit near-death experiences than those without (OR 2.85; Cl 95\% 1.68-4.88; $p=0.0001$ ).

DISCUSSION: Using a crowd-sourcing approach, we found a prevalence of near-death experiences of $10 \%$. While age, gender, place of residence, employment status and perceived threat do not seem to influence the prevalence of near-death experiences, we confirmed a possible association with REM sleep intrusion. 
3

4

5

6

7

\author{
2 Prevalence of near-death experiences in people with and without REM sleep intrusion \\ Daniel Kondziella ${ }^{1-3^{*}}$, Jens P. Dreier ${ }^{4-8}$, Markus Harboe Olsen ${ }^{9}$ \\ ${ }^{1}$ Department of Neurology, Rigshospitalet, University of Copenhagen, Copenhagen, Denmark \\ ${ }^{2}$ Faculty of Health Sciences and Medicine, Copenhagen University, Copenhagen, Denmark \\ ${ }^{3}$ Department of Neuroscience, Norwegian University of Science and Technology, Trondheim, Norway \\ ${ }^{4}$ Center for Stroke Research Berlin, Charité - Universitätsmedizin Berlin, corporate member of Freie \\ Universität Berlin, Humboldt-Universität zu Berlin, and Berlin Institute of Health, Berlin, Germany \\ ${ }^{5}$ Department of Neurology, Charité - Universitätsmedizin Berlin, corporate member of Freie Universität \\ Berlin, Humboldt-Universität zu Berlin, and Berlin Institute of Health, Berlin, Germany \\ ${ }^{6}$ Department of Experimental Neurology, Charité - Universitätsmedizin Berlin, corporate member of \\ Freie Universität Berlin, Humboldt-Universität zu Berlin, and Berlin Institute of Health, Berlin, Germany \\ ${ }^{7}$ Bernstein Center for Computational Neuroscience Berlin, Berlin, Germany \\ ${ }^{8}$ Einstein Center for Neurosciences Berlin, Berlin, Germany \\ ${ }^{9}$ Department of Neuroanesthesiology, Rigshospitalet, University of Copenhagen, Copenhagen, Denmark \\ * Corresponding author: $\quad$ Daniel Kondziella, MD, MSc, dr.philos., FEBN \\ Department of Neurology, \\ Rigshospitalet, Copenhagen University Hospital \\ Blegdamsvej 9 \\ DK-2100 Copenhagen \\ daniel_kondziella@yahoo.com
}




\section{Abstract}

30 BACKGROUND: The origin and prevalence of near-death experiences are unknown. A recent study 31 suggested a link with REM sleep intrusion but was criticized for its selection of control participants. We 32 therefore assessed the association of REM intrusion and near-death experiences with different methods.

METHODS: Using a crowd-sourcing platform, we recruited 1034 lay people from 35 countries to investigate the prevalence of near-death experiences and self-reported REM sleep intrusion. Reports were validated using the Greyson Near-Death Experiences Scale (GNDES) with $\geq 7$ points as cut-off for neardeath experiences.

RESULTS: Near-death experiences were reported by 106 of 1034 participants (10\%; $\mathrm{Cl} 95 \%$ 8.5-12\%). Evidence of REM intrusion was more common in people with near-death experiences ( $n=50 / 106 ; 47 \%)$ than in people with experiences with 6 points or less on the GNDES $(n=47 / 183 ; 26 \%)$ or in those without such experiences ( $n=107 / 744 ; 14 \% ; p=<0.0001$ ). Following multivariate regression analysis to adjust for age, gender, place of residence, employment and perceived danger, this association remained highly significant; people with REM intrusion were more likely to exhibit near-death experiences than those without (OR 2.85; Cl 95\% 1.68-4.88; $p=0.0001$ ).

DISCUSSION: Using a crowd-sourcing approach, we found a prevalence of near-death experiences of $10 \%$. While age, gender, place of residence, employment status and perceived threat do not seem to influence the prevalence of near-death experiences, we confirmed a possible association with REM sleep intrusion. 
48

49

50

51

52

53

54

55

56

57

58

59

60

61

62

63

64

65

66

67

68

69

70

71

72

73

74

75

76

77

78

79

80

81

82

83

84

85

86

87

88

\section{Introduction}

Near-death experiences can be defined as conscious perceptual experiences, including emotional, selfrelated, spiritual and mystical experiences, occurring in a person close to death or in situations of imminent physical or emotional threat (Greyson, 1983). Reports of near-death experiences include, but are not limited to, increased speed of thoughts, distortion of time perception, out-of-body experiences, and visual and auditive hallucinations (Greyson, 1983; Knoblauch, Schmied \& Schnettler, 2001; van Lommel et al., 2001; Martial et al., 2017; Cassol et al., 2018). Yet the pathophysiological basis of neardeath experiences remains unknown (Peinkhofer, Dreier \& Kondziella, 2019).

Near-death experiences share phenotypical features with those made during rapid eye movement (REM) sleep (Table 1) (Nelson et al., 2006). REM sleep is defined by rapid and random saccadic eye movements, loss of muscle tone, a propensity towards vivid dreams, and cortical activation as revealed by EEG desynchronization (Peever \& Fuller, 2017). Importantly, REM state features can intrude into wakefulness, both in healthy people and those with narcolepsy, which may lead to visual and auditory hallucinations at sleep onset (hypnagogic) or upon awakening (hypnopompic) and muscle atonia with sleep paralysis and cataplexy (Scammell, 2015; Jalal \& Ramachandran, 2017; Baird et al., 2018).

In a recent case-control study, people with near-death experiences reported significantly more often REM intrusion than age- and sex-matched controls, and the authors suggested that REM sleep intrusion might contribute to near-death experiences (Nelson et al., 2006). However, the study was criticized for several reasons, including selection bias (Long \& JM, 2007). From a registry of 446 self-reported near-death experiences, 55 North American participants responded to email inquiry, fulfilled inclusion criteria and were enrolled; in contrast, controls were recruited from medical center personnel or their contacts (Nelson et al., 2006). Critics pointed out that controls might have been influenced by their medical background and that "an ideal comparison group would have been made up of people who had been through life-threatening events comparable to [those with near-death experiences], who matched [those with near-death experiences] on other identifying aspects such as culture; and who were willing to report their experiences on a public website, but who had not had [a near-death experience]" (Long \& JM, 2007).

We therefore examined the association between REM sleep intrusion and near-death experiences using a different approach. We recruited a large multinational sample of unprimed lay people from a crowdsourcing platform (without any other selection criteria than English language and age $\geq 18$ years) to estimate the prevalence of near-death experiences and to test the hypothesis that near-death experiences are associated with a propensity for REM sleep intrusion.

\section{Materials \& Methods}

Hypotheses and research questions

The objectives of this study were two-fold.

Peer) reviewing PDF | (2019:05:37745:2:0:NEW 24 Jul 2019) 
89

90

91

92

93

94

95

96

97

98

99

100

101

102

103

104

105

106

107

108

109

110

111

112

113

114

115

116

117

118

119

120

121

122

123

124

125

126

127

128

129
- Primary objective: To estimate the frequency of near-death experiences and REM sleep intrusions reported in a large sample of adult humans collected from an online crowdsourcing service.

- Secondary objective: To test the hypothesis that people who report a near-death experience have a greater frequency of REM sleep intrusions.

Study design

An online platform, Prolific Academic (https://prolific.ac/), was used to recruit a large global sample of lay people. Prolific Academic is a crowdsourcing online platform to recruit human subjects for research that compares favorably in terms of honesty and diversity of participants and data quality (Woods et al., 2015; Peer et al., 2017). Participants were recruited without any filters except for English language and age $\geq 18$ years.

The survey was announced under the headline "Survey on Near-Death Experiences and Related Experiences". Prior to the start of the survey, participants were instructed that their monetary reward was fixed, regardless of whether they would report having had a near-death experience or not. No further information was given. We then asked participants to complete a questionnaire comprising demographic information on age, gender, employment status and place of residence, followed by 4 questions about REM sleep intrusion (Table 1). The questions about REM sleep intrusion were identical to those used by Nelson et al. in their case-control study (Nelson et al., 2006). Participants were then asked if they ever had had a near-death experience (regardless if this experience had occurred in life-threatening or nonlife-threatening situations). If not, the survey ended here; if yes, we continued to inquire about this experience in detail, including all 16 items of the Greyson Near-Death Experience Scale (GNDES), the most widely used standardized tool to identify, confirm and characterize near-death experiences in research (Greyson, 1983). In addition, we inquired about unpleasant feelings (Table 1), which is not covered by the GNDES. Further, participants were given the possibility to describe their experience in their own words. See Table 1 for details.

Statistics

We estimated the number of participants required to be 384, using a very high population size (300.000.000), a confidence level of $95 \%$ and a margin of error of 5\%. However, as previous studies have estimated that $4-8 \%$ of the population have had a near-death experience (Knoblauch, Schmied \& Schnettler, 2001; Perera M, Padmasekara G, 2005; Facco \& Agrillo, 2012), we decided to enroll approximately 1000 participants to identify an estimated 50-100 individuals with near-death experiences.

Data were analyzed using R (R 3.4.1, R Development Core Team [2008], Vienna, Austria). Categorical variables were analyzed using a chi-squared test. Continuous variables were compared using Student's ttests. We calculated odds ratios for having near-death experiences with or without co-occurrence of REM sleep intrusion and performed multivariate logistic regression analysis to correct for age, gender, employment status, place of residence, and whether the situation in which an experience was made was

Peer) reviewing PDF | (2019:05:37745:2:0:NEW 24 Jul 2019) 
130

131

132

133

134

135

136

137

138

139

140

141

142

143

144

145

146

147

148

149

150

151

152

153

154

155

156

157

158

159

160

161

162

163

164

165

166

167

168

169

170

perceived as life-threatening or not. To adjust for multiple testing, we used Bonferroni correction and set the alpha level at 0.01 .

To prevent HARKing (Hypothesizing After the Results are Known) (Fraser et al., 2018), we pre-registered the study, including all objectives, with the Open Science Framework (https://osf.io/ykr3g).

\section{Ethics}

Participants gave consent for publication of their (anonymous) data. Participation was anonymous, voluntary and restricted to those $\geq 18$ years. Participants received a monetary reward upon completion of the survey, following the platform's ethical rewards principle ( $\geq \$ 6.50 / \mathrm{h})$. The Ethics Committee of the Capital Region of Denmark waives approval for online surveys (Section 14 (1) of the Committee Act. 2; http://www.nvk.dk/english).

Data Availability Statement

All de-identified raw data are provided in the online supplemental files. The data analysis plan can be accessed via the Open Science Framework (https://osf.io/ykr3g).

\section{Results}

We recruited 1034 lay people from 35 countries (mean age 32.7 years, standard deviation 11.3 years; $59 \%$ female; $79 \%$ fully or part-time employed or in training), most of which were residing in Europe and North America. Table 2 and Figure 1 provide epidemiological information.

\section{Near-death experiences: Prevalence and semiology}

Two-hundred eighty-nine participants (28\%; Cl 95\% 25-31\%) claimed to have had a near-death experience, 106 of which reached the threshold of 7 points or more on the GNDES (37\%; CI 95\% 31-43\%). Thus, confirmed near-death experiences were reported by 106/1034 participants (10\%; Cl 95\% 8.5-12\%) (Figure 2).

Participants perceived the situation in which they had their experience slightly more often as truly lifethreatening $(n=158 ; 55 \%)$ than not truly life-threatening $(n=131 ; 45 \%)$, and this was irrespective of whether their experience met criteria for a near-death experience according to the GNDES or not $(p=0.55$; Table 3).

Near-death experiences and experiences with $\leq 6$ points on the GNDES occurred in the following situations, listed with decreasing frequency: motor accident $27 \%(n=77)$, near-drowning $19 \%(n=56)$; intense grief or anxiety $18 \%(n=51)$, substance abuse $11 \%(n=33)$; psychological distress without organic disease $9.7 \%(n=28)$; physical violence other than combat $8.3 \%(n=24)$, critical illness $8.0 \%(n=23)$;

Peer] reviewing PDF | (2019:05:37745:2:0:NEW 24 Jul 2019) 
171 childbirth complication $8.0 \%(n=23)$; suicide attempt $6.9 \%(n=20)$; anesthesia/medical procedure $6.9 \%$ $172(n=20)$; cardiac arrest/heart attack 5.5\% $(n=16)$; meditation or prayer $5.2 \%(n=15)$; anaphylactic reaction $1734.8 \%(n=14)$; combat situation 3.8\% ( $n=11)$; syncope $1.7 \%(n=5)$; epileptic seizure $1.4 \%(n=4)$; and other $17419 \%(n=56)$.

175

The most often reported symptoms were abnormal time perception (faster or slower than normal; reported by 252 participants; 87\%); exceptional speed of thoughts ( $n=189 ; 65 \%$ ); exceptional vivid senses $(n=182 ; 63 \%)$; and feeling separated from one's body, including out-of-body experiences ( $n=152 ; 53 \%)$.

179

Experiences that qualified as a true near-death experience according to the GNDES were perceived much more often as pleasant $(n=41 ; 53 \%)$ than experiences that did not $(n=21 ; 14 \% ; p<0.0001$; chisquared test; neutral experience excluded; Table 3).

183

Around one third of participants reporting a near-death experience or near-death-like experience stated having had two or three such experiences ( $n=92,32 \%)$; and some even claimed to have had more than three $(n=10 ; 3.5 \%)$.

187

Near-death experiences and evidence for REM sleep intrusion

189

Evidence for REM sleep intrusion (i.e. $\geq 3$ criteria fulfilled) was much more common in people with experiences above the cut-off point of the GNDES ( $n=50 / 106 ; 47 \%)$ than in people with experiences below this threshold $(n=47 / 183 ; 26 \%)$ or in those without any such experience $(n=107 / 744 ; 14 \% ; p=<0.0001$; chisquared test; Tables $\mathbf{2}$ and 3). Following multivariate regression analysis to adjust for age, gender, place of origin, employment status and perceived danger, this association remained highly significant; i.e. people with REM sleep intrusion were more likely to exhibit near-death experiences than those without REM sleep abnormalities (odds ratio 2.85; Cl 95\% 1.68-4.88; $p=0.0001$; Table 4).

197

Selected written reports from participants can be found in Tables $\mathbf{5}$ and $\mathbf{6}$. Raw data are provided in the online supplemental files.

200

201

\section{Discussion}

202

203

\section{Prevalence and semiology of near-death experiences}

204

Using crowdsourcing methods, we found that 1 out of 10 people from a large sample of 35 countries had 206 a confirmed near-death experience (10\%; Cl 95\% 8.5-12\%). This estimate is slightly higher than what was reported in previous studies using traditional interview-based surveys in Australia (8\%) (Perera $M$, Padmasekara G, 2005) and Germany (4\%) (Knoblauch, Schmied \& Schnettler, 2001); but, of note, none of those studies validated reports with the GNDES. Experiences that did not fulfill criteria for a near-death experience were roughly twice as common in our survey.

211 
212 Similar to previous reports, we found that near-death experiences occur in various cultures and 213 nationalities and irrespective of employment status, age and gender (Thonnard et al., 2013; Charland-

214 Verville et al., 2015; Martial et al., 2017, 2018; Cassol et al., 2018). However, unlike most previous reports 215 in which near-death experiences were almost always associated with peacefulness and well-being 216 (Thonnard et al., 2013; Charland-Verville et al., 2015; Martial et al., 2017, 2018; Cassol et al., 2018), we 217 found a much higher rate of people stating that their experience was unpleasant. Although experiences 218 with a cut-off score of at least 7 points on the GNDES were more often pleasant (53\%) than experiences 219 with a lower score (14\%; $p<0.0001)$, almost half of all near-death experiences were labelled as stressful. 220 It should be noted that Thonnard et al., 2013, Charland-Verville et al., 2015 and Cassol et al., 2018 included

221

222

223

224

225

226

227

228

229

230

231

232

233

234

235

236

237

238

239

240

241

242

243

244

245

246

247

248

249

250

251

252

253 participants who had experienced near-death experiences in life-threatening situations only, i.e. excluding experiences made in non-life-threatening circumstances, which might have led to different frequencies of reported positive and negative emotions. In addition, however, we think the discrepancy between the present and many previous studies is likely because the GNDES addresses only pleasant feelings but does not include negative emotions, in contrast to our questionnaire (Greyson, 1983). Indeed, in line with our results, a recent study revealed a rather high proportion of negative near-death experiences (14\%) (Cassol et al., 2019). Interestingly, and in accordance with our assumptions, that study also included near-death experiences of different etiologies and showed a higher proportion of suicidal attempts among people with negative near-death experiences (Cassol et al., 2019).

Again unlike previous studies (Thonnard et al., 2013; Charland-Verville et al., 2015; Martial et al., 2017, 2018; Cassol et al., 2018), we found that near-death experiences occurred equally likely in truly lifethreatening situations and situations that only just felt so. This discrepancy probably results from the fact that we interviewed unprimed lay people from a large cross-sectional sample, whereas previous studies were retro- or prospectively performed in specific populations such as cardiac arrest survivors (van Lommel et al., 2001; Cassol et al., 2018). This substantiates previous reasoning that near-death experiences are real experiences and not merely products of fantasy proneness (Martial et al., 2018): People with confirmed near-death experiences (i.e. GNDES score $\geq 7$ points) did not perceive their situations as more dangerous than those whose experience did not qualify as a near-death experience (i.e. GNDES score $\leq 6$ points), which argues against tendencies towards overdramatizing.

\section{Near-death experiences and REM sleep intrusion}

Our central finding is that we confirmed a possible association of near-death experiences with REM sleep intrusion. Following multivariate analysis, REM sleep intrusion was the only factor that remained significantly correlated with near-death experiences (and indeed very much so: $p=0.0001$ ). This finding corroborates and extends data from the previous study by Nelson and co-workers, in which the life-time prevalence of REM sleep intrusion in 55 humans with near-death experiences was compared with that in age- and sex-matched controls (Nelson et al., 2006). Sleep-related visual and auditory hallucinations and/or sleep paralysis assessed by a questionnaire like the one used in our study were substantially more common in cases with near-death experiences. The authors suggested that under circumstances of peril, near-death experiences are more likely in people with a tendency towards REM sleep intrusion and that REM sleep intrusion might explain much, if not all, of the semiology of these experiences (Nelson et al.,

Peer) reviewing PDF | (2019:05:37745:2:0:NEW 24 Jul 2019) 
254

255

256

257

258

259

260

261

262

263

264

265

266

267

268

269

270

271

272

273

274

275

276

277

278

279

280

281

282

283

284

285

286

287

288

289

290

291

292

293

294

295
2006). Indeed, as shown in Table 5, two participants from our study gave spontaneous reports of classic REM sleep disturbances (rather than reporting their near-death experience as requested) akin to those seen in people with narcolepsy (Kondziella \& Arlien-Soborg, 2006; Scammell, 2015).

Nelson et al. based the hypothesis of REM intrusion being associated with near-death experiences on several "lines of evidence": REM intrusion during wakefulness occurs frequently in healthy people (from $1.2-32 \%$ for cataplexy to $24-28 \%$ for hypnagogic hallucinations (Ohayon et al., 1999; Cheyne, Rueffer \& Newby-Clark, 1999); REM intrusion is the hallmark of narcolepsy that shares semiological features with near-death experiences; and cardiorespiratory afferents may evoke REM intrusion by heightened vagal afferent activity (Nelson et al., 2006). Further, complex dream-like hallucinations like those of REM sleep are well-described with lesions near the mesopontine paramedian reticular formation and the midbrain cerebral peduncles (i.e. peduncular hallucinations) (Galetta \& Prasad, 2017), suggesting that dysfunction of the REM-inhibiting serotonergic dorsal raphe nuclei and the noradrenergic locus ceruleus may facilitate REM intrusion (Hobson, McCarley \& Wyzinski, 1975; Manford \& Andermann, 1998; Kayama \& Koyama, 2003; de Lecea, Carter \& Adamantidis, 2012; Hasegawa et al., 2017). Nelson et al. therefore hypothesized that people with near-death experiences may have an arousal system predisposing them to REM intrusion (Nelson et al., 2006).

The anonymous nature of our online survey enabled us to avoid the selection bias that was a major point of criticism (Long \& JM, 2007) of the Nelson et al. study (Nelson et al., 2006). While the latter used a casecontrol approach, we evaluated an unselected sample of unprimed adult lay people. We carefully adjusted for confounding factors including age, gender, place of residence, employment status and perceived danger, and we found that the association of near-death experiences and evidence for REM intrusion remained statistically highly significant. Of note, online surveys limit the influence of psychological bias as compared to face-to-face interviews or telephone surveys because there is no incentive to please the investigator by inventing or exaggerating memories (Peer et al., 2017). (There was no monetary incentive to do so either, since we instructed participants that their reimbursement was the same irrespective of whether they would report a near-death experience or not). Also, we recruited a much larger sample size than what can be achieved with lab-based behavioral testing or case-control studies, including respondents from 35 countries, which strengthens the validity and generalizability of our results. It therefore appears that the association of near-death experiences and REM intrusion is real, although future work needs to address the relationship between the two states: Does a tendency to REM intrusion predispose to near-death experiences, as Nelson et al. argue (Nelson et al., 2006), or could it be the other way round, i.e. do near-death experiences lead to a propensity for REM sleep intrusion (Long \& JM, 2007)? Either way, we suggest that characterizing the precise biological mechanisms leading to REM intrusion into wakefulness might offer new insights into the physiology of near-death experiences.

\section{Limitations}

Despite the advantages outlined above, online studies have limitations that should be acknowledged (Woods et al., 2015; Peer et al., 2017). First, complex clinical and ethical notions are impossible to fully implement in a survey form. For instance, almost a third of our participants claimed to have had two or

Peer] reviewing PDF | (2019:05:37745:2:0:NEW 24 Jul 2019) 


\section{Conclusions}

323

324

325

326

327

328

329

330

331

332

333

334 more near-death experiences or near-death-like experiences, but we do not know if these participants only referred to one experience when completing the GNDES or several, which might have skewed GNDES scores in some cases. Second, although we assessed various demographic factors, there are many with potential importance such as religiosity (Greyson, 2006; Chandradasa et al., 2018) that we did not record. (Although we did assess religiosity in a previous online survey using the same crowdsourcing platform and found that most participants have a secular background (Kondziella, D.; Cheung, M.C..; Dutta, 2019)). We did neither inquire about medical history (e.g., diagnoses of epilepsy, narcolepsy, sleep deprivation and migraine), medication or use of alcohol and illicit drugs. Third, for obvious (and ethical) reasons participants were informed about the topic of the survey, which could have led to a selection bias by evoking the interest of survey participants with near-death experiences (and hence overestimation of the true prevalence of such experiences). However, as stated above, the prevalence rate in our study is only slightly higher than that reported in earlier studies (Knoblauch, Schmied \& Schnettler, 2001; Perera M, Padmasekara G, 2005; Facco \& Agrillo, 2012), and the entire survey was finished during such a short time frame (i.e. the number of required participants was reached within 3.5h) that word-of-mouth communication of the survey's topic appears very unlikely. Also, REM sleep intrusion was assessed using a self-reported questionnaire, the sensitivity and specificity of which remains undefined, and objective sleep testing might have yielded different results. However, sleep studies including polysomnography to confirm reports of REM intrusion would have been impractical in such a large multinational sample, and our questionnaire was identical to that from Nelson et al. (Nelson et al., 2006), reflecting screening for REM sleep intrusion as performed by neurologists in clinical practice (Kondziella \& Arlien-Soborg, 2006; DelRosso, Chesson \& Hoque, 2013; Scammell, 2015; Schneider \& Mignot, 2017). Lastly, it should be noted that we did not inquire about the frequency of REM intrusion or the temporality of near-death experiences and REM intrusion. Thus, we do not know which of them occurred first; and if there is a causal relationship, the direction remains unknown, i.e. REM sleep intrusion might predispose to or be induced by near-death experiences.

In our study, the prevalence of near-death experiences was around 10\%. Using a large intercontinental sample of lay people assessed by crowd-sourcing methods, we replicated findings from an earlier casecontrol study suggesting an association of near-death experiences with REM sleep intrusion (Nelson et al., 2006). Thus, whereas age, gender, place of residence, employment status and factual danger of the situation do not appear to influence the frequency with which near-death experiences occur, there seems to be a significant association with REM sleep intrusion. Although association is not causality, identifying the physiological mechanisms behind REM intrusion into wakefulness might advance our understanding of near-death experiences. 
335

336

337

338

339

340

341

342

343

344

345

346

347

348

349

350

351

352

353

354

355

356

357

358

359

360

361

362

363

364

365

366

367

368

369

370

371

372

373

374

375

376

377

\section{References}

Baird B., Castelnovo A., Gosseries O., Tononi G. 2018. Frequent lucid dreaming associated with increased functional connectivity between frontopolar cortex and temporoparietal association areas. Scientific Reports 8:17798. DOI: 10.1038/s41598-018-36190-w.

Cassol H., Martial C., Annen J., Martens G., Charland-Verville V., Majerus S., Laureys S. 2019. A systematic analysis of distressing near-death experience accounts. Memory (Hove, England):1-8. DOI: 10.1080/09658211.2019.1626438.

Cassol H., Pétré B., Degrange S., Martial C., Charland-Verville V., Lallier F., Bragard I., Guillaume M., Laureys S. 2018. Qualitative thematic analysis of the phenomenology of near-death experiences. PLOS ONE 13:e0193001. DOI: 10.1371/journal.pone.0193001.

Chandradasa M., Wijesinghe C., Kuruppuarachchi KALA., Perera M. 2018. Near-Death Experiences in a Multi-religious Hospital Population in Sri Lanka. Journal of Religion and Health 57:1599-1605. DOI: 10.1007/s10943-017-0442-9.

Charland-Verville V., Lugo Z., Jourdan J-P., Donneau A-F., Laureys S. 2015. Near-Death Experiences in patients with locked-in syndrome: Not always a blissful journey. Consciousness and Cognition 34:28-32. DOI: 10.1016/j.concog.2015.03.011.

Cheyne JA., Rueffer SD., Newby-Clark IR. 1999. Hypnagogic and Hypnopompic Hallucinations during Sleep Paralysis: Neurological and Cultural Construction of the Night-Mare. Consciousness and Cognition 8:319-337. DOI: 10.1006/ccog.1999.0404.

DelRosso LM., Chesson AL., Hoque R. 2013. Characterization of REM Sleep without Atonia in Patients with Narcolepsy and Idiopathic Hypersomnia using AASM Scoring Manual Criteria. Journal of Clinical Sleep Medicine 9:675-80. DOI: 10.5664/jcsm.2836.

Facco E., Agrillo C. 2012. Near-death experiences between science and prejudice. Frontiers in Human Neuroscience 6:209. DOI: 10.3389/fnhum.2012.00209.

Fraser H., Parker T., Nakagawa S., Barnett A., Fidler F. 2018. Questionable research practices in ecology and evolution. PLOS ONE 13:e0200303. DOI: 10.1371/journal.pone.0200303.

Galetta KM., Prasad S. 2017. Historical Trends in the Diagnosis of Peduncular Hallucinosis. Journal of Neuro-Ophthalmology 38:1. DOI: 10.1097/WNO.0000000000000599.

Greyson B. 1983. The near-death experience scale. Construction, reliability, and validity. The Journal of nervous and mental disease 171:369-75.

Greyson B. 2006. Near-death experiences and spirituality. Journal of Religious Sciences 41:393414.

Hasegawa E., Maejima T., Yoshida T., Masseck OA., Herlitze S., Yoshioka M., Sakurai T., Mieda M. 2017. Serotonin neurons in the dorsal raphe mediate the anticataplectic action of orexin neurons by reducing amygdala activity. Proceedings of the National Academy of Sciences of the United States of America 114:E3526-E3535. DOI: 10.1073/pnas.1614552114.

Hobson JA., McCarley RW., Wyzinski PW. 1975. Sleep cycle oscillation: reciprocal discharge by two brainstem neuronal groups. Science (New York, N.Y.) 189:55-8.

Jalal B., Ramachandran VS. 2017. Sleep Paralysis, "The Ghostly Bedroom Intruder"; and Out-ofBody Experiences: The Role of Mirror Neurons. Frontiers in human neuroscience 11:92. DOI: 10.3389/fnhum.2017.00092. 
378

379

380

381

382

383

384

385

386

387

388

389

390

391

392

393

394

395

396

397

398

399

400

401

402

403

404

405

406

407

408

409

410

411

412

413

414

415

416

417

418

419

420

421

Kayama Y., Koyama Y. 2003. Control of sleep and wakefulness by brainstem monoaminergic and cholinergic neurons. Acta neurochirurgica. Supplement 87:3-6.

Knoblauch H., Schmied I., Schnettler B. 2001. Different Kinds of Near-Death Experience: A Report on a Survey of Near-Death Experiences in Germany. Journal of Near-Death Studies 20:15-29.

Kondziella, D.; Cheung, M.C..; Dutta A. 2019. Public Perception of the Vegetative State/Unresponsive Wakefulness Syndrome: A Crowdsourced Study. PeerJ in press.

Kondziella D., Arlien-Soborg P. 2006. Diagnostic and therapeutic challenges in narcolepsyrelated psychosis. DOI: 10.4088/JCP.v67n1122b.

de Lecea L., Carter ME., Adamantidis A. 2012. Shining Light on Wakefulness and Arousal. Biological Psychiatry 71:1046-1052. DOI: 10.1016/j.biopsych.2012.01.032.

van Lommel P., van Wees R., Meyers V., Elfferich I. 2001. Near-death experience in survivors of cardiac arrest: a prospective study in the Netherlands. The Lancet 358:2039-2045. DOI: 10.1016/S0140-6736(01)07100-8.

Long J., JM H. 2007. Does the Arousal System Contribute to Near-Death and Out-of-Body Experiences? A Summary and Response. Journal of Near-Death Studies 25:135-169.

Manford M., Andermann F. 1998. Complex visual hallucinations. Clinical and neurobiological insights. Brain : a journal of neurology 121 ( Pt 10):1819-40.

Martial C., Cassol H., Antonopoulos G., Charlier T., Heros J., Donneau A-F., Charland-Verville V., Laureys S. 2017. Temporality of Features in Near-Death Experience Narratives. Frontiers in Human Neuroscience 11:311. DOI: 10.3389/fnhum.2017.00311.

Martial C., Cassol H., Charland-Verville V., Merckelbach H., Laureys S. 2018. Fantasy Proneness Correlates With the Intensity of Near-Death Experience. Frontiers in Psychiatry 9:190. DOI: 10.3389/fpsyt.2018.00190.

Nelson KR., Mattingly M., Lee SA., Schmitt FA. 2006. Does the arousal system contribute to near death experience? Neurology 66:1003-1009. DOI: 10.1212/01.wnl.0000204296.15607.37.

Ohayon MM., Zulley J., Guilleminault C., Smirne S. 1999. Prevalence and pathologic associations of sleep paralysis in the general population. Neurology 52:1194-200. DOI: 10.1212/wnl.52.6.1194.

Peer E., Brandimarte L., Samat S., Acquisti A. 2017. Beyond the Turk: Alternative platforms for crowdsourcing behavioral research. Journal of Experimental Social Psychology 70:153-163. DOI: 10.1016/j.jesp.2017.01.006.

Peever J., Fuller PM. 2017. The Biology of REM Sleep. Current Biology 27:R1237-R1248. DOI: 10.1016/j.cub.2017.10.026.

Peinkhofer C., Dreier J., Kondziella D. 2019. Semiology and mechanisms of near-death experiences. Current Neurology and Neuroscience Reports in press.

Perera M, Padmasekara G BJ. 2005. Prevalence of Near-Death Experiences in Australia. Journal of Near-Death Studies 24:109-116.

Scammell TE. 2015. Narcolepsy. New England Journal of Medicine 373:2654-2662. DOI: 10.1056/NEJMra1500587.

Schneider L., Mignot E. 2017. Diagnosis and Management of Narcolepsy. Seminars in Neurology 37:446-460. DOI: 10.1055/s-0037-1605554.

Thonnard M., Charland-Verville V., Brédart S., Dehon H., Ledoux D., Laureys S., Vanhaudenhuyse A. 2013. Characteristics of Near-Death Experiences Memories as 
422 Compared to Real and Imagined Events Memories. PLoS ONE 8:e57620. DOI:

423 10.1371/journal.pone.0057620.

424 Woods AT., Velasco C., Levitan CA., Wan X., Spence C. 2015. Conducting perception research 425 over the internet: a tutorial review. PeerJ 3:e1058. DOI: 10.7717/peerj.1058.

426 


\section{Table $\mathbf{1}$ (on next page)}

Questionnaire on REM sleep intrusion and near-death experiences.

Questionnaire on REM sleep intrusion and near-death experiences. REM - rapid eye movements; * in contrast to the Near-Death Experience Scale, we also inquired about unpleasant experiences 
1 Table 1. Questionnaire on REM sleep intrusion and near-death experiences. REM - rapid eye movements;

$2 *$ in contrast to the Near-Death Experience Scale, we also inquired about unpleasant experiences

Questions about REM sleep intrusion (1 point for each positive answer; based on Nelson et al., 2006 (Nelson et al., 2006))

- Just before falling asleep or just after awakening, have you ever seen objects, things or people that others can't see?

- Just before falling asleep or just after awakening, have you ever heard voices, music or sounds that other people can't hear?

- Have you ever awakened and felt paralyzed or found that you were unable to move?

- Have you ever had abrupt muscle weakness in your legs or knee buckling, or felt sudden muscle weakness in your face or head drop?

Questions about near-death experiences

- Near-death experiences can be defined as any conscious perceptual experience, including emotional, self-related, spiritual and/or mystical experiences, occurring in a person close to death or in situations of intense physical or emotional danger. In plain language - near-death experiences are exceptional experiences that you may have when you are dying or feel as if you were dying. Have you ever had such a near-death experience - either during a true lifethreatening event or an event that just felt so?

- Was your near-death experience associated with a true life-threatening event or an event that was not life-threatening but felt so?

- In which situation did you have a near-death experience?

- Have you had more than 1 near-death experience?

- If you wish, please describe your experience as detailed as you can (optional). We are interested to know what you felt and how your experience unfolded over time.

Greyson Near-Death Experience Scale (0-2 points for each answer; based on Greyson, 1983 (Greyson, 1983))

- Did time seem to speed up or slow down?

- Were your thoughts speeded up?

- Did scenes from your past come back to you?

- Did you suddenly seem to understand everything?

- Did you have a feeling of peace or pleasantness? *

- Did you have a feeling of joy?

- Did you feel a sense of harmony or unity with the universe?

- Did you see, or feel surrounded by, a brilliant light?

- Were your senses more vivid than usual?

- Did you seem to be aware of things going on elsewhere, as if by extrasensory perception or telepathy?

- Did scenes from the future come to you?

- Did you feel separated from your body?

- Did you seem to enter some other, unearthly world?

- Did you seem to encounter a mystical being or presence or hear an unidentifiable voice?

- Did you see deceased or religious spirits?

- Did you come to a border or point of no return? 


\section{Table 2 (on next page)}

Demographic data and prevalence of REM sleep intrusion.

To adjust for multiple testing, the alpha level was set to 0.01 . Significant $p$ values are shown in bold script. $\mathrm{N}$ - number of participants; NDE - near-death experiences; REM - rapid eye movements; SD - standard deviation; * when comparing "No NDE" $(n=744)$ with confirmed near-death experiences with a Greyson NDE Scale score $\geq 7(n=106$; see Table 3), this significance is lost ( $p$-value $=0.256)$. 
1 Table 2

2 Demographic data and prevalence of REM sleep intrusion. To adjust for multiple testing, the alpha level 3 was set to 0.01 . Significant $p$ values are shown in bold script. $\mathrm{N}$ - number of participants; NDE - near4 death experiences; REM - rapid eye movements; SD - standard deviation; * when comparing "No NDE" $5 \quad(n=744)$ with confirmed near-death experiences with a Greyson NDE Scale score $\geq 7$ ( $n=106$; see Table 3), 6 this significance is lost ( $p$-value $=0.256)$.

\begin{tabular}{|l|r|r|r|r|}
\hline & $\begin{array}{c}\text { All } \\
(\mathrm{n}=1034)\end{array}$ & $\begin{array}{c}\text { No NDE } \\
(\mathrm{n}=744)\end{array}$ & $\begin{array}{r}\text { All claimed NDE } \\
(\mathrm{n}=289)\end{array}$ & $p$-value \\
\hline Age - mean \pm SD & $32.7 \pm 11.3$ & $33.4 \pm 11.3$ & $30.8 \pm 11.1$ & $\mathbf{0 . 0 0 0 6 *}$ \\
\hline Gender $-\mathrm{n}(\%)$ & & & & $\mathbf{0 . 0 0 2}$ \\
\hline Female & $607(59 \%)$ & $461(62 \%)$ & $145(50 \%)$ & \\
\hline Male & $424(41 \%)$ & $282(38 \%)$ & $142(49 \%)$ & \\
\hline Other & $3(0.3 \%)$ & $1(0.1 \%)$ & $2(0.7 \%)$ & \\
\hline Continent - $\mathrm{n}(\%)$ & & & & 0.03 \\
\hline Americas & $36(3.5 \%)$ & $19(2.6 \%)$ & $17(5.9 \%)$ & \\
\hline Asia & $13(1.3 \%)$ & $8(1.1 \%)$ & $4(1.4 \%)$ & \\
\hline Europe & $938(91 \%)$ & $687(92 \%)$ & $251(87 \%)$ & \\
\hline Oceania & $19(1.8 \%)$ & $14(1.9 \%)$ & $5(1.7 \%)$ & \\
\hline Other & $28(2.7 \%)$ & $16(2.2 \%)$ & $12(4.2 \%)$ & \\
\hline Work- $\mathrm{n}(\%)$ & & & & 0.34 \\
\hline Full-Time & $393(38 \%)$ & $281(38 \%)$ & $112(39 \%)$ & \\
\hline Job seeking & $57(5.5 \%)$ & $41(5.5 \%)$ & $15(5.2 \%)$ & \\
\hline Not in paid work & $99(9.6 \%)$ & $75(10 \%)$ & $24(8.3 \%)$ & \\
\hline Part-Time & $217(21 \%)$ & $166(22 \%)$ & $51(18 \%)$ & \\
\hline Student & $174(17 \%)$ & $116(16 \%)$ & $58(20 \%)$ & \\
\hline Other & $94(9.1 \%)$ & $65(8.7 \%)$ & $29(10 \%)$ & \\
\hline REM intrusion $-\mathrm{n}(\%)$ & & & & $<0.0001$ \\
\hline$\leq 2$ criteria & $829(80 \%)$ & $637(86 \%)$ & $192(66 \%)$ & \\
\hline$\geq 3$ criteria & $204(20 \%)$ & $107(14 \%)$ & $97(34 \%)$ & \\
\hline
\end{tabular}




\section{Table 3 (on next page)}

Participants claiming a near-death experience, analyzed according to Greyson NearDeath Experience Scale score.

A score of $\geq 7$ confirms the reported experience as a near-death experience. IQR interquartile range; $\mathrm{n}$ - number of participants; NDE - near-death experience(s); REM - rapid eye movements; SD - standard deviation; significant $p$-values are shown in bold script; *excluding participants reporting that their experience was neither pleasant nor unpleasant 
1 Table 3

2 Participants claiming a near-death experience, analyzed according to Greyson Near-Death Experience 3 Scale score. A score of $\geq 7$ confirms the reported experience as a near-death experience. IQR 4 interquartile range; $\mathrm{n}$ - number of participants; NDE - near-death experience(s); REM - rapid eye 5 movements; SD - standard deviation; significant $\mathrm{p}$-values are shown in bold script; * excluding participants 6 reporting that their experience was neither pleasant nor unpleasant

\begin{tabular}{|c|c|c|c|c|}
\hline & $\begin{array}{l}\text { All claimed NDE } \\
\quad(n=289)\end{array}$ & $\begin{array}{c}\text { Greyson NDE score }<7 \\
\qquad(n=183)\end{array}$ & $\begin{array}{c}\text { Greyson NDE score } \geq 7 \\
(n=106)\end{array}$ & $p$-value \\
\hline Greyson NDE score - median (IQR) & $5(3-8)$ & $4(3-5)$ & $9(8-14)$ & \\
\hline Age - mean \pm SD & $30.8 \pm 11.1$ & $30.0 \pm 10.7$ & $32.0 \pm 11.6$ & 0.14 \\
\hline Gender - n (\%) & & & & 0.34 \\
\hline Female & $145(50 \%)$ & $98(54 \%)$ & $47(44 \%)$ & \\
\hline Male & $142(49 \%)$ & $84(46 \%)$ & $58(55 \%)$ & \\
\hline Other & $2(0.7 \%)$ & $1(0.5 \%)$ & $1(0.9 \%)$ & \\
\hline REM intrusion $-\mathrm{n}(\%)$ & & & & 0.0003 \\
\hline$\leq 2$ criteria & $192(66 \%)$ & $136(74 \%)$ & $56(53 \%)$ & \\
\hline$\geq 3$ criteria & $97(34 \%)$ & $47(26 \%)$ & $50(47 \%)$ & \\
\hline Life-threatening event $-\mathrm{n}(\%)$ & & & & 0.55 \\
\hline Yes & $158(55 \%)$ & $103(56 \%)$ & $55(52 \%)$ & \\
\hline No & $131(45 \%)$ & $80(44 \%)$ & $51(48 \%)$ & \\
\hline Feelings associated with NDE $*-n(\%)$ & $(n=230)$ & $(n=153)$ & $(n=77)$ & $<0.0001$ \\
\hline Unpleasant & $168(73 \%)$ & $132(86 \%)$ & $36(47 \%)$ & \\
\hline Pleasant & $62(27 \%)$ & $21(14 \%)$ & $41(53 \%)$ & \\
\hline
\end{tabular}




\section{Table 4 (on next page)}

Multivariate logistic regression and odds ratios for having a near-death experience (Greyson Near-Death Experience Scale $\geq 7$ ).

To adjust for multiple testing, the alpha level was set to 0.01 . $\mathrm{Cl}$ - confidence interval; $\mathrm{n}$ number of participants; OR - odds ratio; REM - rapid eye movements; significant $p$-values are shown in bold script 
1 Table 4

2 Multivariate logistic regression and odds ratios for having a near-death experience (Greyson Near-Death

3 Experience Scale $\geq 7$ ). To adjust for multiple testing, the alpha level was set to 0.01 . $\mathrm{Cl}$ - confidence 4 interval; OR - odds ratio; REM - rapid eye movements; significant $p$-values are shown in bold script

\begin{tabular}{|l|l|l|}
\hline & OR (Cl 95\%) & $\begin{array}{l}\text { - } \\
\text { value }\end{array}$ \\
\hline Age & $1.01(0.99-1.04)$ & 0.35 \\
\hline Gender & & \\
\hline Female (reference) & 1.00 & \\
\hline Male & $1.58(0.93-2.69)$ & 0.09 \\
\hline Other & $1.73(0.05-59.89)$ & 0.74 \\
\hline Work & & \\
\hline Full-Time (reference) & 1.00 & \\
\hline Part-Time & $0.47(0.22-0.99)$ & 0.05 \\
\hline Job seeking & $0.59(0.16-1.93)$ & 0.40 \\
\hline Not in paid work & $0.96(0.35-2.58)$ & 0.94 \\
\hline Student & $0.54(0.25-1.15)$ & 0.11 \\
\hline Other & $0.45(0.15-1.3)$ & 0.15 \\
\hline Continent & & \\
\hline Americas (reference) & 1.00 & \\
\hline Asia & $0(0-\mathrm{NA})$ & 0.98 \\
\hline Europe & $0.37(0.12-1.05)$ & 0.06 \\
\hline Oceania & $0.59(0.05-5.79)$ & 0.65 \\
\hline Other & $0.49(0.08-2.92)$ & 0.43 \\
\hline REM intrusions & & \\
\hline$\leq 2$ criteria (reference) & 1.00 & \\
\hline$\geq 3$ criteria & $2.85(1.68-4.88)$ & 0.0001 \\
\hline Life-threatening event & & \\
\hline No (reference) & 1.00 & 0.53 \\
\hline Yes & $0.85(0.5-1.43)$ & \\
\hline & & \\
\hline
\end{tabular}




\section{Table 5 (on next page)}

Selected reports from participants with an experience that reached the threshold of $\geq 7$ points on the Greyson NDE scale to qualify as a near-death experience.

Note that the last two comments describe instances that are highly suggestive of REM sleep disturbance, including visual hypnogogic hallucinations and sleep paralysis, rather than the near-death experience both participants reported to have had. Comments are edited for clarity and spelling. 


\section{Table 5}

Selected reports from participants with an experience that reached the threshold of $\geq 7$ points on the Greyson NDE scale to qualify as a near-death experience. Note that the last two comments describe instances that are highly suggestive of REM sleep disturbance, including visual hypnogogic hallucinations and sleep paralysis, rather than the near-death experience both participants reported to have had. Comments are edited for clarity and spelling.

- I was at the beach in Florida, I was 10-11. Suddenly, huge waves started pulling me further and further from the shore. As I was fighting, my life started flashing before me in my head. [...] I felt like my soul was being pulled out of my body. I was floating and was [lifted in the air]. After a few moments I felt like I was in an enormous tunnel of darkness, and at its end there was the brightest white light I have ever seen. I remember that my dead relatives were at the gate, including my maternal grandmother. I don't remember what we talked about. But then I felt that I was sucked out of the tunnel and I fell, crashing into my body again. Male, 28 years; near-drowning; Greyson NDE Scale score 10; fulfilling 3 of 4 criteria for REM sleep intrusion

- I encountered a truly out-of-body experience where my eyesight and visual became incredibly abstract. For around an hour I had no sense of self or my surroundings. When my self-awareness returned, I became concerned that I was indeed dying or had died. I eventually became completely lucid, and still to this day I do not understand this experience. Male, 46 years; drug intoxication; Greyson NDE scale 8; REM 3/4

- I was very young when I almost drowned. I saw angels, and they were singing the most beautiful music I have ever heard. I was very upset when I was revived. Female, 57 years; near-drowning; Greyson NDE scale 15; REM 1/4

- During my first cardiorespiratory arrest I was aware of being outside my body. My partner saw me at the window, calling for help, but at this point I was not breathing. Female, 35 years; critical illness/cardiac attack; Greyson NDE scale 25; REM 3/4

- I felt like I just died, and I went to heaven. I heard voices, and I was sure I would not come back to my life. It was weird. I could not control my body. Female, 37 years; childbirth; Greyson NDE scale 11; REM $3 / 4$

- It was a very pleasant experience: Intense white light, feelings of overwhelming love. I had a sense of not having done all the things I was meant to do. I heard a nurse repeatedly calling my name and telling me to breathe. I eventually took a breath. It was a very positive experience and has affected my whole life since in a very positive way. Female, 59 years; childbirth complication; Greyson NDE scale 15; REM 2/4

- I nearly drowned when I was around 8 years old. I felt total peace. Twenty years later I can still remember how I felt. It was an amazing feeling. Female, 32 years; near-drowning; Greyson NDE scale 7; REM 2/4

- I often see characters in my hallway or feel someone else's presence before going to sleep. Male, 32 years; near-drowning; Greyson NDE scale 11, REM 4/4

- Sometimes I wake at night, and I can't move. I see strange things, like spirits or demons at my door, and after a while I see them coming beside me. I can't move or talk, and they sit on my chest. It scares the hell out of me! I think that it is a dream, count to 3 and close my eyes. Sometimes this helps. Female, 28 years; physical violence; NDE 20; REM 4/4 


\section{Table 6(on next page)}

Selected reports from participants with an experience below the threshold of $\geq 7$ points on the Greyson NDE scale.

Comments are edited for clarity and spelling. 


\section{Table 6}

3 Selected reports from participants with an experience below the threshold of $\geq 7$ points on the Greyson

4 NDE scale. Comments are edited for clarity and spelling.

- I felt extreme fear and was certain I would die. At one point I suddenly stopped resting against what was certain to come, and instead a feeling of complete calm and acceptance came over me. I was fully in the moment and had no thoughts of anything else. When I was out of danger, I was shaken but the memory of the "good feeling" was clear. Female, 50 years; psychological distress without organic disease; Greyson NDE Scale score 3; fulfilling 0 of 4 criteria for REM sleep intrusion

- I hit the back of my head on a swimming pool springboard. I remember seeing myself outside of my body being helped, while I was unconscious. Male, 32 years; concussion; Greyson NDE scale 4, REM 2/4

- During a fire evacuation of an 18-story building, I apparently slept through and didn't evacuate. However, I experienced myself in peace, floating in the hallways and watching the other residents evacuate the building. Talking to them in the following days I could describe who passed me, and what they took with them or were wearing. Male, 46 years; fire evacuation; Greyson NDE scale 6, REM 1/4

- I lost a lot of blood during my last childbirth. I felt floaty and weird as if I was about to leave my body. I didn't feel like I was there anymore. Female, 24 years; childbirth complication; Greyson NDE scale 5; REM 2/4 
Figure 1

Map showing places of residency of survey participants.

Using an online crowdsourcing platform, we recruited 1.034 lay people from 35 countries on 4 continents, the majority from Europe and North America

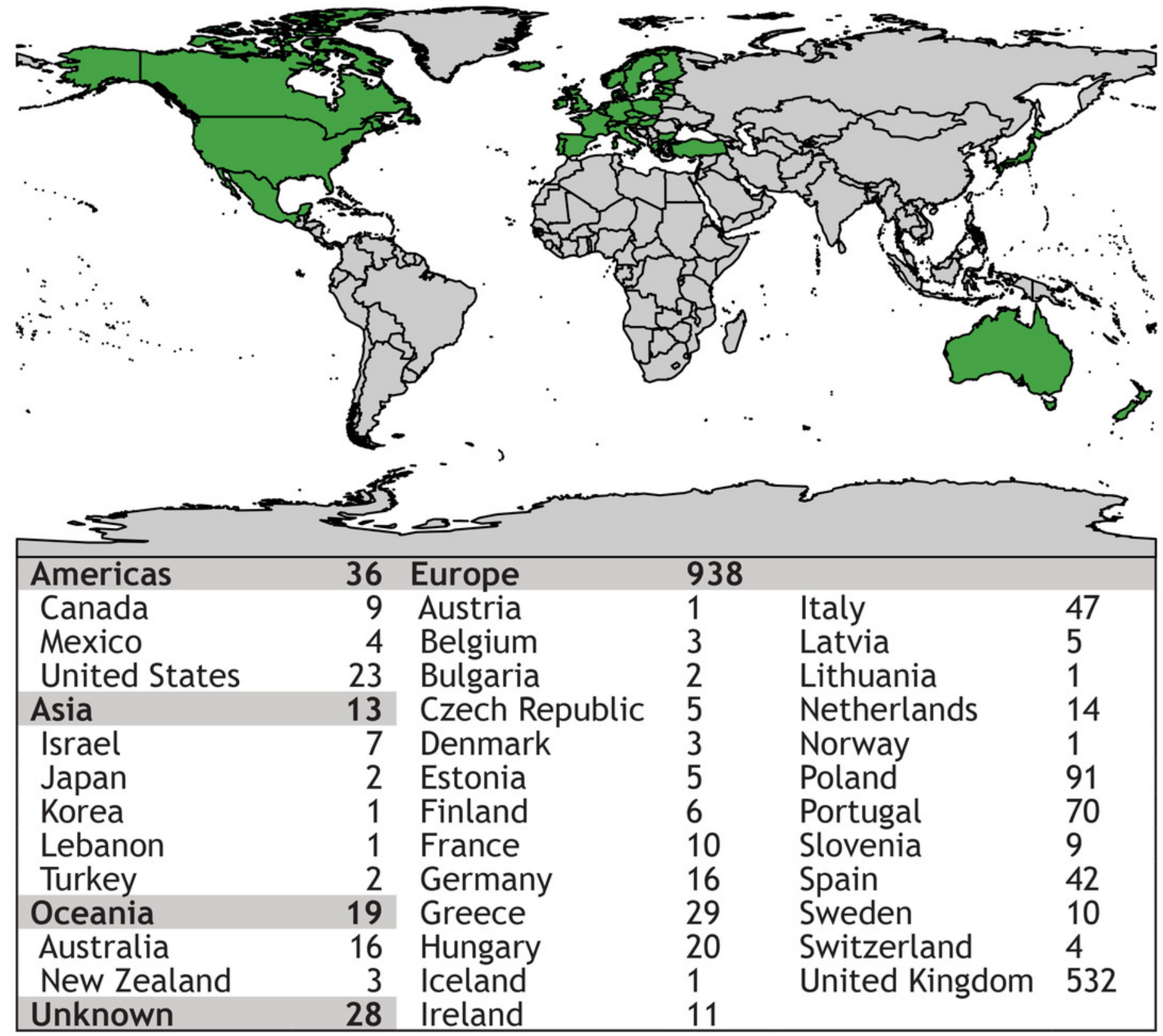


Figure 2

Schematic overview of study design.

Of 61.719 eligible lay people registered with Prolific Academic ( https://prolific.ac/ ; accessed on January 22, 2019), we enrolled 1.034 participants; 106 (10\%; Cl95\%8.5-12\%) of whom reported a near-death experience that fulfilled established criteria (Greyson Near-Death Experience Scale score of 7 or higher). $\mathrm{N}$ = number of participants; NDE - near-death experience 


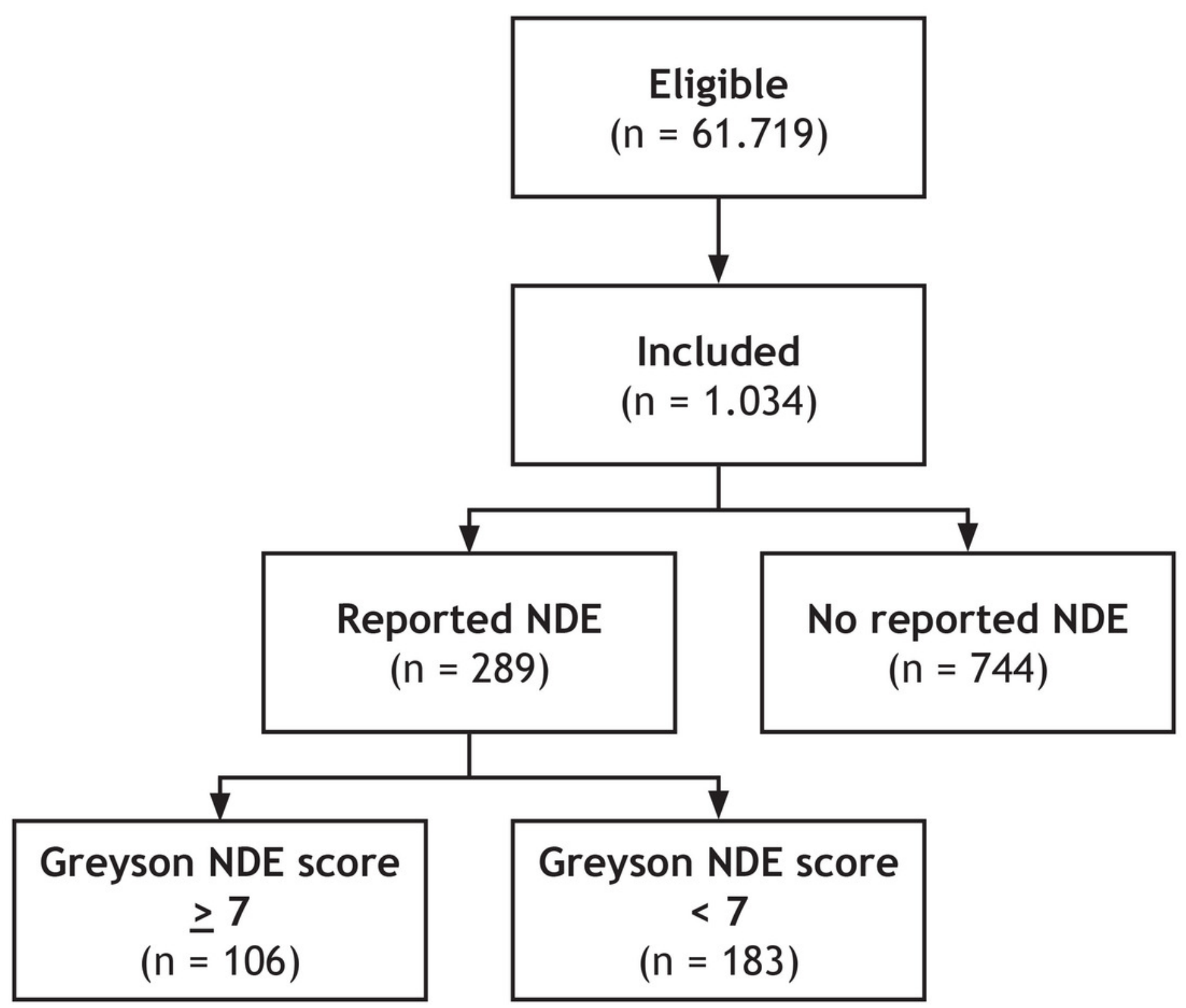

